ЧАСТОТА НАЗНАЧЕНИЯ И ПРИВЕРЖЕННОСТЬ ЛЕЧЕНИЮ СТАТИНАМИ

У АМБУЛАТОРНЫХ БОЛЬНЫХ САХАРНЫМ ДИАБЕТОМ 2 ТИПА С КОМОРБИДНОЙ СЕРДЕЧНО-СОСУДИСТОЙ ПАТОЛОГИЕЙ

(с) В.Н. Серебрякова*, Е.А. Головина, А.В. Кавешников, В.С. Кавешников

Научно-исследовательский институт кардиологии, Томский национальный исследовательский медицинский центр Российской академии наук, Томск

ОБОСНОВАНИЕ. В связи с высоким темпом роста заболеваемости и бремени сердечно-сосудистых осложнений сахарный диабет 2 типа (СД2) является значимой медицинской проблемой в мире. Даже при отсутствии сердечно-сосудистых заболеваний (СС3) пациенты с СД2 относятся к категории высокого и очень высокого риска. Кроме контроля гликемии, крайне важным аспектом ведения данной группы пациентов является профилактика сердечно-сосудистых осложнений. Наличие СД2 и гиперлипидемии определяет целевую группу для приема статинов. Вместе с тем опубликовано недостаточно информации о частоте приема данного класса лекарств среди лиц с СД2.

ЦЕЛЬ. Изучить частоту назначения статинов и приверженность лечению у амбулаторных больных с СД2 с коморбидной сердечно-сосудистой патологией.

МЕтоДЫ. Обследовано 156 пациентов с СД2 (87,2\% - женщины, средний возраст - 65,2 года) в рамках амбулаторного приема врача-эндокринолога городской поликлиники г. Томска. Использовали стандартный опросник, составленный на основе адаптированных международных методик, включающий информацию о кардиальной патологии, принимаемых препаратах, уровне дохода, тест Мориски-Грина. Измеряли антропометрические показатели, уровень глюкозы плазмы натощак, гликированного гемоглобина, показателей липидного спектра. Для сравнения использовали методы параметрической и непараметрической статистики.

РЕЗУЛЬТАТЫ. По данным исследования, статины назначены 45,0\% обследуемых, постоянно принимали - 47,0\% из них. В 41 и 39\% случаев статины назначались эндокринологом и кардиологом соответственно. Принимающие статины пациенты характеризовались более тяжелым функциональным классом стенокардии (p=0,03), более высокой частотой перенесенного инфаркта миокарда ( $\mathrm{p}=0,01)$. По другим сопутствующим заболеваниям, показателям углеводного обмена различий между группами не выявлено. Треть пациентов были привержены (3-4 балла), 2/3 не привержены лечению (0-2 балла) соответственно. Пациенты с доходом более 1 и менее 2 прожиточных минимумов принимали статины чаще $(p=0,021)$.

ЗАКЛючЕНИЕ. Выявлена недостаточная частота назначения и приверженность терапии статинами у пациентов с СД2. В большинстве случаев статины назначались эндокринологом или кардиологом. Функциональный класс стенокардии, перенесенный инфаркт миокарда и умеренный доход ассоциировались с более частым приемом статинов. Для повышения охвата лечением статинами лиц с СД2 большего внимания требуют вопросы профилактики СС3 как со стороны медицинских специалистов, так и пациентов.

КЛЮЧЕВЫЕ СЛОВА: сахарный диабет; сердечно-сосудистые заболевания; статины; частота назначения; приверженность лечению; коморбидность

\title{
PRESCRIBING FREQUENCY AND ADHERENCE TO STATINS IN OUTPATIENTS WITH TYPE 2 DIABETES MELLITUS AND COMORBID CARDIOVASCULAR DISEASES
}

\author{
(c) Victoria N. Serebryakova*, Elena A. Golovina, Artem V. Kaveshnikov, Vladimir S. Kaveshnikov
}

Cardiology Research Institute, Tomsk National Research Medical Center, Russian Academy of Sciences, Tomsk, Russia

BACKGROUND: Due to the high rate of growth in the incidence and burden of cardiovascular complications, type 2 diabetes mellitus (T2DM) is a significant medical problem in the world. Even in the absence of cardiovascular disease (CVD), patients with T2DM are classified as high and very high risk. In addition to glycemic control, an extremely important aspect of managing this group of patients is prevention of cardiovascular complications. T2DM and hyperlipidemia determines the target group for statins. At the same time, little is known about the frequency of administration of this class of drugs among people with T2DM.

AIM: To study prescribing frequency and adherence to statins in outpatients with T2DM and comorbid cardiovascular diseases. 
METHODS: 156 patients with type 2 diabetes (87.2\% - women, average age - 65.2 years) were examined as part of an outpatient appointment with an endocrinologist at the city polyclinic of Tomsk. We used a standard questionnaire compiled on the basis of adapted international methods, including information on cardiac pathology, medications, income level, and Morisky-Green test. Anthropometric parameters, fasting plasma glucose, glycated hemoglobin, lipid spectrum parameters were measured. Methods of parametric and nonparametric statistics were used for comparisons.

RESULTS: Statins were prescribed to $45.0 \%$ of the surveyed, and $47.0 \%$ of them were constantly taking statins. In 41 and $39 \%$ of cases, statins were prescribed by an endocrinologist and a cardiologist, respectively. Those taking statins were characterized by a more severe functional class of angina pectoris $(p=0.03)$, a higher prior myocardial infarction rate $(p=0.01)$. For other concomitant diseases, and also indicators of carbohydrate metabolism, differences between the groups were not revealed. One third of patients were adherent (3-4 points), 2/3 were not adherent to treatment (0-2 points), respectively. Patients with incomes between 1 and 2 cost of living took statins more often than the rest $(p=0.021)$.

CONCLUSION: An insufficient frequency of prescription and adherence to statin therapy in patients with T2DM was revealed. In most cases, statins were prescribed by an endocrinologist or cardiologist. Functional class of angina pectoris, prior myocardial infarction and moderate income were associated with more frequent use of statins. To increase the coverage of patients with T2DM with statin treatment, more attention needs to be paid to the issues of CVD prevention from both medical professionals and patients.

KEYWORDS: diabetes mellitus; cardiovascular disease; statins; prescription frequency; adherence to treatment; comorbidity

Сердечно-сосудистые заболевания (ССЗ) и сахарный диабет 2 типа (СД2) - достаточно распространенные заболевания, нередко сочетающиеся между собой. Основной причиной смертности среди пациентов с СД2 являются ССЗ. В свою очередь, СД2 является независимым фактором риска развития и прогрессирования сердечно-сосудистой патологии, которая встречается в среднем в 2-4 раза чаще, чем в популяции пациентов без диабета. С учетом увеличения продолжительности жизни населения, распространения ожирения и гиподинамии будет по-прежнему наблюдаться рост заболеваемости СД, преимущественно за счет СД2 [1-3].

Пациенты с СС3, имеющие СД2, относятся к группе пациентов с неблагоприятным прогнозом. Давно доказано, что данная группа пациентов характеризуется высоким риском сердечно-сосудистых осложнений. Так, у пациентов с СД2 риск развития инсульта и инфаркта миокарда в 2-6 раз выше, а выживаемость после острых сердечно-сосудистых событий в 2-3 раза ниже [4, 5].

В связи с этим к данной коморбидной патологии всегда отмечался высокий интерес, особенно у пациентов старшей возрастной группы. Взаимоотягощающее и нередко атипичное течение способны приводить к более ранним осложнениям $[2,6]$.

На этом фоне основной тенденцией в лечении данной коморбидной патологии остается комплексный подход, цель которого - не только коррекция гипергликемии, но и других факторов риска, прежде всего артериальной гипертензии (АГ) и дислипидемии. Такой подход ведет к снижению прогрессирования ССЗ и частоты осложнений у данной категории пациентов [7]. В связи с этим весьма актуальным остается вопрос о назначении терапии с доказанной эффективностью в отношении сердечно-сосудистых событий, способной благоприятно влиять на прогноз. Одной из таких групп препаратов являются ингибиторы ГМГ-КоА-редуктазы (статины), рекомендованные к применению как для вторичной, так и для первичной профилактики ССЗ у пациентов высокого и очень высокого сердечно-сосудистого риска $[8,9]$.

Однако по-прежнему сохраняются сложности ведения таких групп пациентов в практическом здравоох- ранении, особенно на амбулаторном этапе. Несмотря на достижения современной медицины в области профилактики и лечения СС3, современные эпидемиологические исследования демонстрируют крайне недостаточный прием статинов среди лиц с СД даже в группе очень высокого риска СС3 [10]. Другой проблемой является то, что достижение целевых уровней корригируемых метаболических параметров не происходит в достаточной мере. Многие пациенты демонстрируют неспособность длительно соблюдать рекомендации в отношении образа жизни и регулярно принимать лекарственные препараты, что является важной медицинской проблемой, ведущей к неблагоприятным последствиям [11].

\section{ЦЕЛЬ}

Изучить частоту назначения статинов и приверженность лечению у амбулаторных больных с СД2 с коморбидной сердечно-сосудистой патологией.

\section{МЕТОДЫ}

\section{Дизайн исследования}

Наблюдательное одноцентровое ретроспективное исследование.

\section{Критерии соответствия}

В исследование включены пациенты с СД2, диагноз которым был установлен в соответствии с критериями современной классификации и согласно клиническим рекомендациям. В исследование не включались пациенты с СД 1 типа, не подписавшие добровольное информированное согласие на участие в исследовании, не пожелавшие заполнить анкету и лица с интеллектуально-мнестическими заболеваниями.

\section{Условия проведения}

Исследование проводилось на базе городской поликлиники г. Томска и включало пациентов, обратившихся за амбулаторной помощью к врачу-эндокринологу. Все 
обследованные подписывали добровольное информированное согласие на участие в исследовании.

\section{Продолжительность исследования}

Исследование продолжалось с 2019 по 2020 гг. и включало данные, полученные на момент обращения за амбулаторной помощью.

\section{Описание медицинского вмешательства}

На приеме всем пациентам было предложено заполнить вопросник, состоящий из нескольких разделов. Первый раздел включал паспортную часть, где фиксировались пол, возраст и уровень образования. Второй раздел содержал информацию о сопутствующей кардиальной патологии: АГ, ишемической болезни сердца (ИБС), перенесенном ранее инфаркте миокарда (ИМ), хронической сердечной недостаточности (ХCH) и проводимых хирургических вмешательствах на сердце (стентирование, коронарное шунтирование, протезирование клапанов сердца). В третьем разделе пациентам было предложено ответить на вопрос о принимаемых регулярно лекарственных препаратах, и была проведена оценка приверженности лечению с использованием теста Мориски-Грина.

На приеме у всех пациентов оценивались антропометрические показатели - рост, вес, индекс массы тела (ИМТ), объем талии (ОТ). По данным амбулаторной карты фиксировались показатели глюкозы плазмы натощак, гликированного гемоглобина $\left(\mathrm{HbA}_{1 c}\right)$, липидного спектра, а также проводился анализ назначенной лекарственной терапии.

\section{Основной исход исследования}

В ходе исследования изучены социально-демографические характеристики, сопутствующая кардиальная патология, параметры углеводного и липидного обмена, терапия статинами и приверженность лечению.

\section{Анализ в подгруппах}

Все обследованные пациенты были разделены на две группы. Группы формировались в зависимости от приема или неприема статинов. В группах была проведена сравнительная оценка основных исследуемых показателей.

\section{Методы регистрации исходов}

Данные о поле, возрасте, уровне образования, сопутствующей кардиальной патологии, принимаемых лекарственных препаратах, уровне дохода на одного члена семьи вносились непосредственно пациентами при заполнении анкет. Ответы на вопросы теста МорискиГрина также заполнялись непосредственно пациентами. Пропущенные данные не замещались.

Рост измерялся с помощью медицинского ростомера РЭП. Для измерения массы тела использовались медицинские весы ВМЭН-150-50/100, прошедшие метрологический контроль. Индекс массы тела рассчитывался путем деления массы тела (в килограммах) на квадрат роста (в метрах квадратных). Измерение ОТ проводилось с использованием стандартной гибкой сантиметровой измерительной ленты. Состояние углеводного и липидного обмена исследовали с помощью стандартных клинико-биохимических методов. Выраженность расстройств углеводного обмена у пациентов с СД2 оце- нивали по содержанию $\mathrm{HbA}_{1 c}$ и глюкозы плазмы натощак. Состояние липидного обмена оценивалось по концентрации общего холестерина (ОХС), липопротеидов низкой плотности (ЛПНП), триглицеридов (ТГ) и липопротеидов высокой плотности (ЛПВП).

\section{Этическая экспертиза}

Исследование одобрено ЛЭК НИИ кардиологии Томского НИМЦ № 187 от 10.07.2019. «Комитет по биомедицинской этике НИИ кардиологии Томского НИМЦ».

\section{Статистический анализ}

Принципы расчета размера выборки: размер выборки предварительно не рассчитывался.

Методы статистического анализа данных: статистическая обработка первичного материала проводилась с помощью пакета программ SPSS (v.13). Для сравнения частот использовали критерий $X^{2}$ или точный тест Фишера. Для сравнения независимых наблюдений большинства непрерывных величин использовали тест Стьюдента, для триглицеридов - тест Манна-Уитни соответственно. Различия считались статистически значимыми при $\mathrm{p}<0,05$.

\section{РЕЗУЛЬТАТЫ}

\section{Объекты (участники) исследования}

Обследовано 156 пациентов: 87,2\% - женщины,

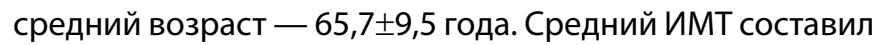
31,95 кг/м². Среди обследованных пациентов у 85,3\% отмечалась АГ, у 11,5\% - ИБС, у 11,5\% - ХСН соответственно. У всех лиц с ИБС фиксировалась АГ. У 5,1\% пациентов в анамнезе отмечался перенесенный ИМ, у 3,2\% - сердечно-сосудистые вмешательства. Общее и абдоминальное ожирение зафиксировано у $48 \%$ и $63,5 \%$ обследованных соответственно; тревога/депрессия - 31,4\%.

\section{Основные результаты исследования}

По данным изучения амбулаторных карт статины были назначены в 45,0\% случаев, из которых постоянный прием статинов отмечался в 47,0\% случаев, что в целом составило 21,0\% всех обследованных пациентов. В $64 \%$ случаев назначался аторвастатин, в $36 \%$ - розувастатин. Изучение вопроса, специалистами какого профиля на амбулаторном этапе впервые были назначены статины (рис. 1), показало, что чаще всего препарат назначался эндокринологом, кардиологом, значительно реже терапевтом. В 7,2\% случаев информация о назначении статинов не была отражена в амбулаторных картах, тогда как в анкетах пациенты отмечали прием данной группы препаратов. При более детальном анализе медицинской документации отмечено, что в 4,3\% из них информация нашла отражение в стационарных выписках, в 2,9\% случаев установить, кем назначена данная группа препаратов, не удалось.

Изучение оценки приверженности лечению в нашем исследовании выявило, что по результатам теста Мориски-Грина 1/3 пациентов были привержены лечению (3-4 балла по шкале), у 2/3 пациентов отсутствовала приверженность лечению (0-2 балла) (рис. 2). Различий в приверженности между исследуемыми группами найдено не было. 


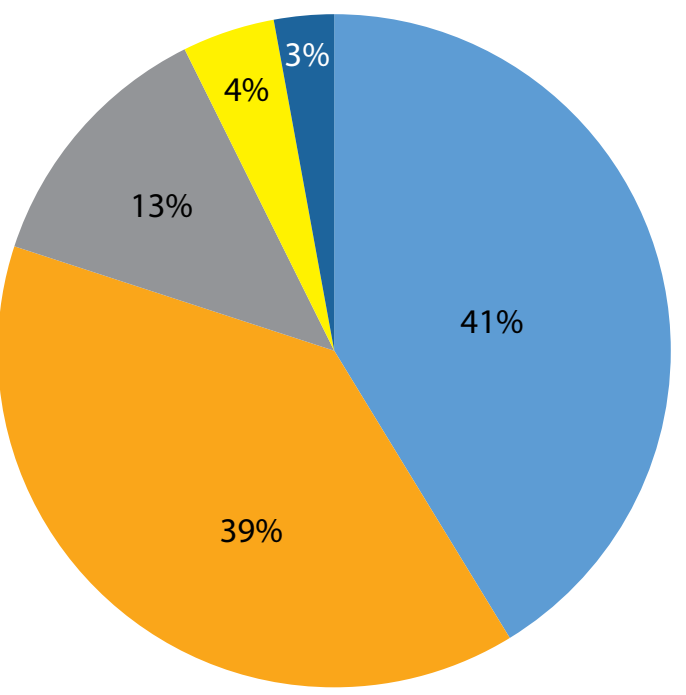

Эндокринолог

Кардиолог

Выписка из стационара

Неизвестно

Терапевт

Рис. 1. Частота назначения статинов специалистами различного профиля.

В зависимости от приема статинов обследованные пациенты были разделены на две группы, клиническая характеристика которых представлена в табл. 1. Как видно из таблицы, средний возраст принявших участие в исследовании пациентов, а также ИМТ между группами статистически значимо не различались. Тяжелый функциональный класс стенокардии напряжения (III-IV) и перенесенный ИМ значительно чаще встречались в группе пациентов, принимающих статины. По сопутствующим заболеваниям, таким как ожирение, АГ, ХСН, сердечно-сосудистым вмешательствам, индикаторам углеводного и липидного обмена статистически значимых различий между группами выявлено не было. Более низкие показатели ОХС и лПНП в группе принимающих статины наблюдались лишь на уровне тенденции (табл. 1).

Данные о частоте приема статинов в зависимости от уровня дохода представлены в табл. 2. Реже других статины принимали при уровне дохода, примерно равном 1 прожиточному минимуму (ПМ), в то время как чаще всего - при умеренном доходе (выше 1 ПМ и ниже 2 ПМ). Одинаково часто прием статинов отмечался при крайних категориях дохода.

\section{ОБСУЖДЕНИЕ}

\section{Резюме основного результата исследования}

Кроме контроля гликемии, крайне важным аспектом ведения пациентов с СД2 является профилактика сердечно-сосудистых осложнений, частота встречаемости которых выше, а последствия серьезнее по сравнению с популяцией без данной патологии. Наличие СД2 и гиперлипидемии определяет целевую группу для приема статинов. В то же время в современной литературе недостаточно освещается вопрос о частоте приема данного класса лекарств среди лиц с СД2.

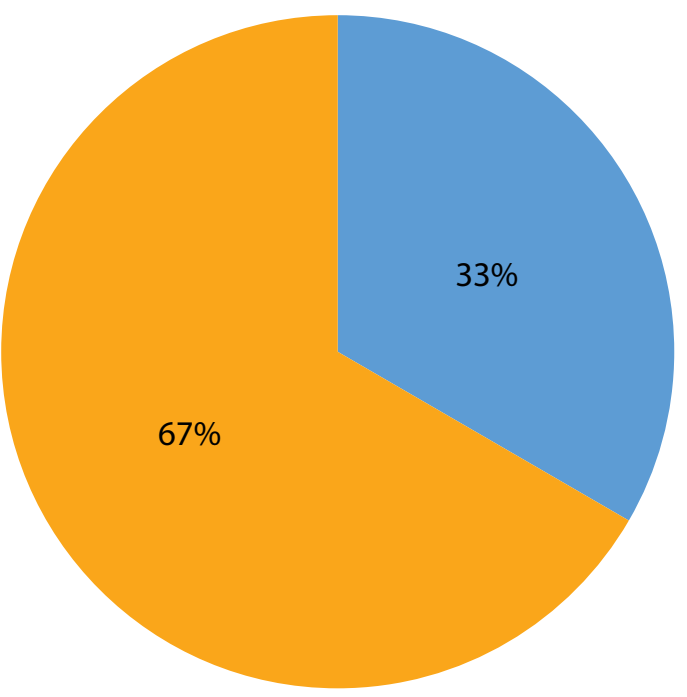

Привержены лечению (3-4 балла)

Не привержены лечению (0-2 балла)

Рис. 2. Приверженность лечению по данным теста Мориски-Грина.

Согласно полученным данным, выявлены недостаточная частота назначения и приверженность терапии статинами у пациентов с СД2. В большинстве случаев статины назначались эндокринологом или кардиологом. Функциональный класс стенокардии, перенесенный ИМ и умеренный доход ассоциировались с более частым приемом статинов.

\section{Обсуждение основного результата исследования}

Согласно полученным данным, частота назначения статинов пациентам с СД2 составила менее 50\%, из них менее 50\% пациентов осуществляли прием лекарства, таким образом, охвачены лечением были только $21 \%$ из имеющих показания пациентов. Несмотря на весомую доказательную базу, демонстрирующую пользу статинов как для первичной, так и вторичной профилактики СС3, охват лечением целевой группы населения остается недостаточным [12]. Из эпидемиологических исследований известно, что наличие СД2 повышает вероятность приема статинов в общей популяции $[13,14]$. В то же время, по данным исследования ЭССЕ-РФ, частота приема данного класса препаратов в российской популяции среди лиц с СД и дополнительными факторами риска (ФР) (очень высокий риск) не превышала 12-13\% [10].

Полученные результаты подтверждают тревожные тенденции, отмечаемые в ряде ранее опубликованных исследований и регистров. Так, исследование АРГО, проводимое в РФ с 2013 по 2014 гг., имеющее своей целью изучить уровень ОХС у амбулаторных пациентов высокого и очень высокого сердечно-сосудистого риска, показало, что уровень ОХС > 5 ммоль/л регистрировался в среднем у 70-80\% обследованных пациентов в различных федеральных округах. Половине пациентов высокого и очень высокого риска не были назначены статины [15]. Позже исследование АЙСБЕРГ [16] показало сопоставимые результаты, свидетельствующие о том, что ситуация с назначением и приемом статинов на амбулаторном этапе существенно не меняется. 
Таблица 1. Клинико-анамнестическая характеристика обследованных пациентов

\begin{tabular}{|c|c|c|c|c|c|c|c|}
\hline \multirow[t]{2}{*}{ Показатель } & \multicolumn{2}{|c|}{$\begin{array}{c}\text { Пациенты, } \\
\text { принимающие } \\
\text { статины }\end{array}$} & \multicolumn{2}{|c|}{$\begin{array}{c}\text { Пациенты, } \\
\text { не принимающие } \\
\text { статины }\end{array}$} & \multicolumn{2}{|c|}{ Bcero } & \multirow[t]{2}{*}{$\mathbf{P}^{3}$} \\
\hline & aбc. & $\%$ & aбc. & $\%$ & абc. & $\%$ & \\
\hline Женщины & 26 & 19,1 & 110 & 80,9 & 136 & 100 & 0,14 \\
\hline Мужчины & 7 & 35,0 & 13 & 65,0 & 20 & 100 & 0,14 \\
\hline Оба пола & 33 & 21,1 & 123 & 78,9 & 156 & 100 & - \\
\hline Возраст, лет ${ }^{1}$ & 65,3 & 8,07 & 65,7 & 9,86 & 65,5 & 9,48 & 0,83 \\
\hline 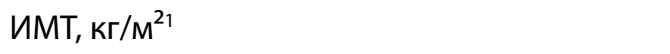 & 31,5 & 4,42 & 32,1 & 4,71 & 31,6 & 4,64 & 0,49 \\
\hline Инфаркт миокарда & 5 & 62,5 & 3 & 37,5 & 8 & 100 & 0,01 \\
\hline Чрескожное коронарное вмешательство & 1 & 50 & 1 & 50 & 2 & 100 & 0,37 \\
\hline Коронарное шунтирование & 1 & 100 & 0 & 0 & 1 & 100 & 0,21 \\
\hline Ожирение (ИМТ $\geq 30$ кг/м²) & 22 & 20,8 & 84 & 79,2 & 106 & 100 & 0,50 \\
\hline $\begin{array}{l}\text { Абдоминальное ожирение } \\
\text { (ОТ } \geq 80 \text { см для женщин } \geq 94 \text { см для мужчин) }\end{array}$ & 23 & 23,2 & 76 & 76,8 & 99 & 100 & 0,4 \\
\hline $\mathrm{A} Г$ & 30 & 22,6 & 103 & 77,4 & 133 & 100 & 0,41 \\
\hline Тревога/депрессия & 12 & 24,4 & 37 & 75,5 & 49 & 100 & 0,48 \\
\hline I-II ФK CH & 3 & 21,4 & 11 & 78,6 & 14 & 100 & 1 \\
\hline III-IV ФK CH & 3 & 75 & 1 & 25 & 4 & 100 & 0,03 \\
\hline I ФК XCH по NYHA & 2 & 40 & 3 & 60 & 5 & 100 & 0,28 \\
\hline II ФК XCH по NYHA & 2 & 25 & 6 & 75 & 8 & 100 & 0,27 \\
\hline III ФК XCH по NYHA & 2 & 40 & 3 & 60 & 5 & 100 & 0,28 \\
\hline IV ФК XCH по NYHA & 0 & 0 & 0 & 0 & 0 & 100 & - \\
\hline Глюкоза, ммоль/л 1 & 8,37 & 3,10 & 8,82 & 2,77 & 8,71 & 2,85 & 0,51 \\
\hline $\mathrm{HbA}_{1 c^{\prime}} \%^{1}$ & 7,40 & 1,82 & 7,24 & 1,38 & 7,27 & 1,49 & 0,59 \\
\hline ОХС, ммоль/л' & 5,23 & 1,54 & 5,90 & 1,46 & 5,74 & 1,50 & 0,054 \\
\hline ЛПНП, ммоль/л'1 & 2,85 & 0,86 & 3,49 & 1,05 & 3,28 & 1,03 & 0,07 \\
\hline ЛПВП, ммоль/л ${ }^{1}$ & 1,29 & 0,30 & 1,34 & 0,40 & 1,33 & 0,37 & 0,684 \\
\hline ТГ, ммоль/л² & 1,50 & $1,1-2,2$ & 2,1 & $1,5-3,0$ & 1,8 & $1,3-2,7$ & 0,116 \\
\hline
\end{tabular}

Примечания. 'Среднее и стандартное отклонение. ${ }^{2}$ Медиана, $25-75 \%$ квартили. ${ }^{3}$ Уровень статистической значимости. Сокращения: АГ - артериальная гипертензия; ИБС — ишемическая болезнь сердца; ИМТ - индекс массы тела; ФК - функциональный класс; ХСН - хроническая сердечная недостаточность; $\mathrm{HbA}_{1 с}$ - гликированный гемоглобин; ОХС — общий холестерин; ЛПНП — липопротеины низкой плотности; ЛПВП — липопротеины высокой плотности; ТГ — триглицериды; ОТ — объем талии.

Таблица 2. Приверженность статинотерапии в зависимости от уровня дохода пациентов

\begin{tabular}{|c|c|c|c|c|c|c|c|}
\hline \multirow{2}{*}{$\begin{array}{l}\text { Уровень } \\
\text { дохода }\end{array}$} & \multicolumn{2}{|c|}{$\begin{array}{c}\text { Пациенты, } \\
\text { принимающие статины }\end{array}$} & \multicolumn{2}{|c|}{$\begin{array}{c}\text { Пациенты, } \\
\text { не принимающие статины }\end{array}$} & \multicolumn{2}{|c|}{ Bcero } & \multirow[t]{2}{*}{$\mathbf{p}$} \\
\hline & aбc. & $\%$ & aбc. & $\%$ & абс. & $\%$ & \\
\hline$<1 \sqcap M$ & 4 & 16,7 & 20 & 83,3 & 24 & 100 & 0,55 \\
\hline$\sim 1 \sqcap \mathrm{M}$ & 3 & 8,8 & 31 & 91,2 & 34 & 100 & 0,046 \\
\hline$>1 \sqcap M$ & 23 & 28,4 & 58 & 71,6 & 81 & 100 & 0,021 \\
\hline$\geq 2 \sqcap M$ & 2 & 15,4 & 11 & 84,6 & 13 & 100 & 0,73 \\
\hline
\end{tabular}

Примечание: ПМ - прожиточный минимум на одного члена семьи. 
Данные ретроспективного амбулаторного регистра РЕКВАЗА показывают, что из числа пациентов, которым показана гиполипидемическая терапия, ее получает, в лучшем случае, каждый третий пациент [8]. После выписки из стационаров многие пациенты принимают рекомендованные препараты какое-то время, но с течением времени приверженность лечению снижается. Согласно данным регистра острого ИМ (Хабаровск), через 2,5 года после выписки из стационара в отношении приема статинов отмечалась наиболее низкая приверженность пациентов, только 24\% принимали соответствующие дозы назначенных препаратов [17]. Более высокие значения исследуемого показателя отмечаются в когортном исследовании Byrne Р. и соавт., по данным которого частота приема статинов в репрезентативной выборке населения Ирландии среди лиц с СД2 составила 57,8\% [13].

Причины недостаточного охвата лечением статинами многогранны и условно могут быть связаны с лечащим врачом, непосредственно с пациентом и/ или системой здравоохранения [18]. Возможными причинами распространенности низкой приверженности К назначению препаратов группы ингибиторов ГМГ-КоА-редуктазы среди врачей могут быть: недостаточное знание клинических рекомендаций, сохраняющаяся распространенность «мифов» о вреде применения статинов среди врачей и, как следствие, назначение диетотерапии пациентам очень высокого сердечно-сосудистого риска [9]. Факторы, влияющие на приверженность лечению со стороны пациентов, могут быть различными, но чаще всего сводятся к слабой мотивации пациентов ввиду отсутствия очевидного эффекта для здоровья при приеме статинов, а также характеристики самого препарата и стоимости лечения [19].

Полученные результаты подтверждают хорошо известную закономерность о более высокой частоте приема статинов среди лиц с ИБС, особенно после перенесенного ИМ $[10,13]$. По мере увеличения функционального класса ИБС и после перенесенного ИМ отмечалось некоторое увеличение как доли назначения статинов, так и роста приверженности лечению $[8,20]$. По данным исследования АЙСБЕРГ, лица, перенесшие реваскуляризацию миокарда, более привержены лечению. Возможно, это связано с более пристальным вниманием врачей и большей обеспокоенностью пациентов состоянием своего здоровья [21]. Но и охват этой группы пациентов, несмотря на определенную тяжесть состояния и коморбидность, остается также неполным [22].

Рассматривая экономические аспекты проблемы низкого охвата лечением статинами, нельзя не затронуть вопрос высокой стоимости оригинальных препаратов. Актуальность данного фактора хорошо демонстрирует улучшение приверженности лечению при снижении стоимости оригинальных препаратов [10]. Научные данные о влиянии социального градиента на вероятность приема статинов довольно неоднозначны. По данным некоторых исследований, пациенты с низким уровнем дохода менее привержены лечению статинами, чем со средним или высоким уровнем дохода [23]. Данная закономерность не находит своего подтверждения в общей популяции [14]. В нашем исследовании среди пациентов, не принимающих статины, и пациентов, принимающих статины, отмечаются значимые различия в уровне дохода, равного или более 1 ПМ на человека в семье, в пользу последних. Таким образом, наиболее высокая частота приема статинов отмечалась среди лиц с доходом выше 1 и менее 2 ПМ.

\section{Ограничения исследования}

Ограничения данного исследования заключаются в небольшом объеме выборки и использовании данных, собранных в одном отдельном учреждении, что может не в полной мере отражать ситуацию на популяционном уровне. Также детально не исследовались вопрос о причине низкого процента назначения статинов специалистами на амбулаторном этапе и причины низкой приверженности пациентов к приему данной группы препаратов.

\section{ЗАКЛЮЧЕНИЕ}

Несмотря на большое количество накопленных данных о пользе статинов для первичной и вторичной профилактики среди пациентов высокого и очень высокого сердечно-сосудистого риска, использование данной группы препаратов среди лиц с СД2 по-прежнему остается недостаточным. Полученные результаты показывают, что данная ситуация складывается как в результате недостаточного назначения препаратов медицинскими специалистами, так и низкой приверженности лечению со стороны пациентов. Подавляющее большинство назначений статинов осуществлено узкими специалистами эндокринологом и кардиологом и лишь малая доля - терапевтами. Полученные данные подтверждают предположение о более высокой частоте приема препаратов данного класса среди лиц с более тяжелой сердечно-сосудистой коморбидной патологией. По-видимому, более частому приему статинов способствует доход более 1 и менее 2 ПМ на 1 человека в семье. Возможно, повлиять на ситуацию могут непрерывные образовательные мероприятия, адресованные вопросам профилактики СС3, не только среди врачей разных специальностей, но и среди пациентов с СД2.

\section{ДОПОЛНИТЕЛЬНАЯ ИНФОРМАЦИЯ}

Источник финансирования. Финансирование исследование проведено за счет бюджетных средств учреждения.

Конфликт интересов. Авторы заявляют об отсутствии явного и потенциального конфликта интересов.

Участие авторов. Серебрякова В.Н. - концепция и дизайн исследования, анализ и интерпретация полученных результатов, анализ литературы, написание текста статьи; Головина Е.А. - сбор и обработка материала, анализ литературы, написание текста статьи; Кавешников А.В. - создание базы данных, обработка, математический анализ полученных данных; Кавешников В.С. - анализ и интерпретация полученных результатов, написание текста статьи. Все авторы внесли существенный вклад в проведение исследования и подготовку статьи, прочли и одобрили финальную версию перед публикацией. 


\section{СПИСОК ЛИТЕРАТУРЫ | REFERENCES}

1. Лукьянов М.М., Концевая А.В., Мырзаматова А.О., и др. Пациенты с сочетанием сердечно-сосудистых заболеваний и сахарного диабета второго типа по данным регистров РЕКВАЗА и РЕГИОН: характеристика мультиморбидности и исходов, оценка потенциального эффекта дапаглифлозина в Российской клинической практике // Рациональная фармакотерапия в кардиологии. - 2020. - Т. 16. — №1. - С. 59-68. [Loukianov MM, Kontsevaya AV, Myrzamatova AO, et al. Patients with combination of cardiovascular diseases and type 2 diabetes in RECVASA and REGION registries: multimorbidity, outcomes and potential effect of dapagliflozin in the Russian clinical practice. Rational Pharmacotherapy in Cardiology. 2020;16(1):59-68. (In Russ.)]. doi: https://doi.org/10.20996/1819-6446-2020-02-03

2. Ворожцова И.Н., Будникова О.В., Афанасьев С.А. Влияние сахарного диабета 2-го типа на миокард пациентов С ишемической болезнью сердца // Сибирский медицинский журнал (2. Томск). — 2018. - Т. 33. — №1. - С. 14-20. [Vorozhtsova IN, Budnikova OV, Afanasyev SA. Influence of type 2 diabetes on th myocardium of patients with ischemic heart disease. The Siberian Medical Journal. 2018;33(1);14-20. (In Russ.)]. doi: https://doi.org/10.29001/2073-8552-2018-33-1-14-20

3. Кузьмичкина М.А., Серебрякова В.Н. Инвалидность и возврат к труду среди пациентов, перенесших хирургическую реваскуляризацию миокарда // Сибирский медииинский журнал. — 2020. - Т. 35. — №2. - C. 44-49. [Kuzmichkina MA, Serebryakova VN. Disability and return to work among patients undergoing surgical myocardial revascularization. The Siberian Medical Journal. 2020;35(2):44-49. (In Russ.)]. doi: https://doi.org/10.29001/2073-8552-2020-35-2-44-49

4. Аметов А.С., Демидова Т.Ю., Кочергина И.И. Эффективность препаратов метформина в лечении сахарного диабета 2-го типа // Медицинский совет. — 2016. — №3. - С. 30-37. [Ametov AS, Demidova TY, Kochergina II. The efficacy of metformin in the treatment of type 2 diabetes. Medical Council. 2016;(3):30-37. (In Russ.)].

5. Богданов А.Н., Добрынина И.Ю., Добрынин Ю.В. Ишемический инсульт при сахарном диабете 2-го типа (обзор литературы) // Наука 21 века: вопросы, гипотезы, ответы. — 2014. — №4. C. 43-52. [Bogdanov AN, Dobryinina IYu, Dobryinin YuV. Nauka 21 veka: voprosy, hipotezy, otvety. 2014;(4):43-52.(In Russ.)].

6. Мелихова С.П., Шевцова В.И., Зуйкова А.А. Изучение коморбидной патологии при сахарном диабете 2 типа как осложнении метаболического синдрома // Архивъ внутренней медииины. — 2018. — Т. 8. — №5. C. 366-371. [Melikhova SP, Shevcova VI, Zujkova AA. Studying of comorbid pathology at the 2 types diabetes as the complication of the metabolic syndrome. The Russian Archives of Internal Medicine. 2018;8(5):366-371. (In Russ.)]. doi: https://doi.org/10.20514/2226-6704-2018-8-5-366-371

7. Сапожникова И.Е., Тарловская Е.И., Ануфриева Е.Н. Лечение сердечно-сосудистых заболеваний у пациентов с сахарным диабетом 2-го типа в реальной клинической практике // Рачиональная фармакотерапия в кардиологии. - 2009. T. 5. — №3. - C. 8-13. [Sapozhnikova IE, Tarlovskaya El, Anufrieva EN. Cardiovascular diseases treatment in patients with type 2 diabetes mellitus in current clinical practice. Rational Pharmacotherapy in Cardiology. 2009;5(3):8-13. (In Russ.)]. doi: https://doi.org/10.20996/1819-6446-2009-5-3-8-13

8. Загребельный А.В., Марцевич С.Ю., Лукьянов М.М., и др. Качество гиполипидемической терапии в амбулаторно-поликлинической практике: данные регистра РЕКВАЗА // Профилактическая медицина. - 2016. - Т. 19. — №1. — C. 9-14. [Zagrebelnyi AV, Martsevich SYu, Lukyanov MM, et al. Quality of lipid-lowering therapy in outpatient practice: recvas register data. The Russian Journal of Preventive Medicine and Public Health. 2016;19(1):9-14. (In Russ.)]. doi: https://doi.org/10.17116/profmed20161919-14

9. Смирнова М.Д., Агеев Ф.Т. Статины - старые мифы и новые факты // РMЖ. - 2017. - T. 25. - №20. - C. 1421-1428. [Smirnova MD, Ageev FT. Statins - the old myths and new facts. Medical Journal of the Russian Federation. 2017;25(20):1421-1428. (In Russ.)]. doi: https://doi.org/10.1016/j.atherosclerosis.2016.11.025

10. Шальнова С.А., Деев А.Д., Метельская В.А., и др.

Информированность и особенности терапии статинами у лиц с различным сердечно-сосудистым риском: исследование ЭССЕ-РФ // Кардиоваскулярная терапия и профилактика. 2016. — T. 15. — №4. - C. 29-37. [Shalnova SA, Deev AD, Metelskaya VA, et al. Awareness and treatment specifics of statin therapy in persons with various cardiovasular risk: the study ESSE-RF. Cardiovascular Therapy and Prevention. 2016;15(4):29-37. (In Russ.)]. doi: https://doi.org/10.15829/1728-8800-2016-4-29-37

11. Лиходей Н.В., Калашникова М.Ф., Лиходей Е.М. Анализ факторов, препятствующих формированию приверженности лечению среди больных сахарным диабетом, и стратегий, способствующих ее повышению // Сахарный диабет. - 2018. - Т. 21. №1. - C. 5-14. [Likhodey NV, Kalashnikova MF, Likhodey EM. Analysis of the factors that prevent adherence to treatment in patients with diabetes mellitus and the strategies that contribute to the improvement in adherence. Diabetes Mellitus. 2018;21(1):5-14. (In Russ.)]. doi: https://doi.org/10.14341/DM8781

12. Карпов Ю.А. Эффективность и безопасность терапии статинами // Атмосфера. Новости кардиологии. - 2019. - № 1. - С. 3-12. [Karpov YuA. The efficacy and safety of statin therapy. Athmosphera novosti kardiologii. 2019;(1):3-12. (In Russ.)].

13. Byrne P, Cullinan J, Murphy C, Smith SM. Cross-sectional analysis of the prevalence and predictors of statin utilisation in Ireland with a focus on primary prevention of cardiovascular disease. BMJ Open. 2018;8(2):e018524. doi: https://doi.org/10.1136/bmjopen-2017-018524

14. Кавешников В.С., Серебрякова В.Н., Трубачева И.А. Частота и предикторы приема статинов в общей популяции трудоспособного возраста // Российский кардиологический журнал. - 2020. - Т. 25. - №6. - C. 102-107. [Kaveshnikov VS, Serebryakova VN, Trubacheva IA. Prevalence and predictors of statin therapy in the general working-age population. Russian Journal of Cardiology. 2020;25(6):102-107. (In Russ.)]. doi: https://doi.org/10.15829/1560-4071-2020-3920

15. Ахмеджанов Н.М., Небиеридзе Д.В., Сафарян А.С., и др. Гиполипидемическая терапия в условиях амбулаторной практики (по данным исследования АРГО-2) // Рациональная фармакотерапия в кардиологии. - 2016. - Т. 12. №2. - C. 147-153. [Akhmedzhanov NM, Nebieridze DV, Safaryan AS, et al. Lipid-lowering therapy in outpatient practice (according to the ARGO-2 study). Rational Pharmacotherapy in Cardiology. 2016;12(2):147-153. (In Russ.)]. doi: https://doi.org/10.20996/1819-6446-2016-12-2-147-153

16. Ахмеджанов Н.М., Небиеридзе Д.В., Сафарян А.С. Коррекция гиперхолестеринемии в первичной и вторичной профилактике сердечно-сосудистых заболеваний: особенности и спорные вопросы // Рачиональная фармакотерапия в кардиологии. - 2018. - Т. 14. - №6. - С. 917-921. [Akhmedzhanov NM, Nebieridze DV, Safaryan AS. Correction of hypercholesterolemia in primary and secondary prevention of cardiovascular diseases: features and controversial issues. Rational Pharmacotherapy in Cardiology. 2018;14(6):917-921. (In Russ.)]. doi: https://doi.org/10.20996/1819-6446-2018-14-6-917-921

17. Малай Л.Н., Давидович И.М. Хабаровский Регистр острого инфаркта миокарда: анализ отдаленных исходов и приверженности длительной медикаментозной терапии // Дальневосточный медичинский журнал. — 2017. — №2. C. 43-48. [Malay LN, Davidovich IM. Khabarovsk register of acute myocardial infarction: the analysis of long-term prognosis and long-term medical treatment compliance. Far Eastern Medical Journal. 2017;(2):43-48. (In Russ.)].

18. Шальнова С.А., Белов В.Н., Валиахметов М.Н., и др. Пути увеличения приверженности терапии статинами // Кардиоваскулярная терапия и профилактика. - 2018. T. 17. — №2. — C. 81-87. [Shalnova SA, Belov VN, Valiakhmetov $\mathrm{MN}$, et al. Approaches to statin therapy adherence improvement. Cardiovascular Therapy and Prevention. 2018;17(2):81-87. (In Russ.)]. doi: https://doi.org/10.15829/1728-8800-2018-2-81-87

19. Боева О.И., Иваненко А.С., Колесников В.Н. Приверженность к терапии статинами в реальной клинической практике // Медицинский вестник Северного Кавказа. - 2010. - №2. C. 26-29. [Boeva OI, Ivanenko AS, Kolesnikov VN. Adherence to statin therapy in the real clinical practice. Medical News of the North Caucasus. 2010;(2):26-29. (In Russ.)]. 
20. Лукьянов М.М., Козминский А.Н., Марцевич С.Ю., и др. Больные с сочетанием хронической сердечной недостаточности, артериальной гипертонии и перенесенного ранее инфаркта миокарда: клинико-анамнестические характеристики и практика назначения ингибиторов ангиотензин-превращающего фермента, блокаторов рецепторов ангиотензина и бета-адреноблокаторов, приверженность лечению (данные амбулаторного регистра РЕКВАЗА) // Рачиональная фармакотерапия в кардиологии. 2017. - T. 13. — №2. - C. 207-212. [Loukianov MM, Kozminsky AN, Martsevich SYu, et al. Patients with combination of chronic heart failure, hypertension and history of myocardial infarction: clinical and anamnestic characteristics, administration of ACE-inhibitors, angiotensin receptor blockers, beta-blockers and adherence to the drug therapy (data of outpatient registry recvasa). Rational Pharmacotherapy in Cardiology. 2017;13(2):207-212. (In Russ.)]. doi: https://doi.org/10.20996/1819-6446-2017-13-2-207-212

21. Загидуллин Н.Ш., Загидуллин Ш.3. Приверженность терапии статинами // Кардиоваскулярная терапия и профилактика. 2008. - T. 7. — №1. - C. 107-111. [Zagidullin NSh, Zagidullin ShZ Statin therapy compliance. Cardiovascular Therapy and Prevention. 2008:7(1):107-111. (In Russ.)].
22. Серебрякова В.Н. Вопросы создания регистра пациентов с ишемической болезнью сердца, подвергшихся операции коронарного шунтирования, на современном этапе развития кардиологической помощи // Сибирский медицинский журнал (2/Томск). - 2019. - Т. 34. - №4. - C. 55-61. [Serebryakova VN. Issues of creating a registry of patients with coronary heart disease undergoing coronary artery bypass surgery at the present stage of cardiological care development. The Siberian Medical Journal. 2019;34(4):55-61. (In Russ.)]. doi: https://doi.org/10.29001/2073-8552-2019-34-4-55-61

23. Гайсенок О.В., Рожков А.Н., Лишута А.С. Гиполипидемическая терапия в аспекте профилактики острых нарушений мозгового кровообращения: существующие стандарты, данные доказательной медицины и реальная практика // Рачиональная фармакотерапия в кардиологии. - 2018. T. 14. - №3. - C. 434-440. [Gaisenok OV, Rozhkov AN, Lishuta AS. Hypolipidemic therapy in stroke prevention: existing standards, evidence-based medicine data and real practice. Rational Pharmacotherapy in Cardiology. 2018;14(3):434-440. (In Russ.)]. doi: https://doi.org/10.20996/1819-6446-2018-14-3-434-440

\section{ИНФОРМАЦИЯ ОБ АВТОРАХ [AUTHORS INFO]}

*Серебрякова Виктория Николаевна, к.м.н. [Victoriya N. Serebryakova, MD, PhD]; адрес: Россия, 634012, Томск, Киевская ул., д. 111A [address: 111A, Kievskaya st., 634012 Tomsk, Russian Federation];

ORCID: https://orcid.org/0000-0002-9265-708X; eLibrary SPIN: 9653-4472; e-mail: vsk75@yandex.ru

Головина Елена Александровна, м.н.c. [Elena A. Golovina, MD, junior research associate]; ORCID: https://orcid.org/0000-0002-3192-2521; eLibrary SPIN: 9001-8129; e-mail: golea_88@mail.ru Кавешников Артем Владимирович, м.н.с. [Artem V. Kaveshnikov, junior research associate]; ORCID: https://orcid.org/0000-0002-4743-1989; eLibrary SPIN: 1646-8539; e-mail: artemkave@mail.ru Кавешников Владимир Сергеевич, к.м.н, в.н.с. [Vladimir S. Kaveshnikov, MD, PhD, leading research associate]; ORCID: https://orcid.org/0000-0002-0211-4525; eLibrary SPIN: 4870-0390; e-mail: kave@ngs.ru

\section{ЦИТИРОВАТЬ:}

Серебрякова В.Н., Головина Е.А., Кавешников А.В., Кавешников В.С. Частота назначения и приверженность лечению статинами у амбулаторных больных сахарным диабетом 2 типа с коморбидной сердечно-сосудистой патологией // Сахарный диабет. — 2020. — Т. 23. — №5. — C. 434-441. doi: https://doi.org/10.14341/DM12563

\section{TO CITE THIS ARTICLE:}

Serebryakova VN, Golovina EA, Kaveshnikov AV, Kaveshnikov VS. Prescribing frequency and adherence to statins in outpatients with type 2 diabetes mellitus and comorbid cardiovascular diseases. Diabetes Mellitus. 2020;23(5):434-441. doi: https://doi.org/10.14341/DM12563 